\title{
CORRELAÇÃO ENTRE TEMPO DE DIAGNÓSTICO, TRATAMENTO E SOBREVIDA EM PACIENTES COM CÂNCER DE MAMA: UMA REVISÃO DE LITERATURA
}

\author{
Gabriel Carlos Leite, Bruna Faust Ruhnke, Fernando Antônio Mourão Valejo \\ Universidade do Oeste Paulista - UNOESTE, Faculdade de Medicina, Presidente Prudente, SP. E-mail: \\ gabriener@gmail.com
}

\begin{abstract}
RESUMO
O câncer de mama é o tumor maligno mais incidente em mulheres no mundo. Trata-se de um importante problema de saúde pública em virtude, principalmente, do seu diagnóstico tardio e, por conseguinte, alta mortalidade. $O$ objetivo foi realizar uma revisão da literatura buscando estudos que correlacionem o tempo transcorrido entre o diagnóstico do câncer de mama e o início do tratamento quimioterápico. Foi realizado um levantamento nas bases de dados: Literatura Latino-Americana e do Caribe em Ciências da Saúde (LILACS), PubMed, Literatura Internacional em Ciências da Saúde (MEDLINE), Scientific Electronic Library Online (SciELO), Sistema de Informação da Biblioteca da OMS (WHOLIS). Foram utilizados os termos "breast cancer" AND "diagnostic time". O tempo de diagnóstico e início do tratamento são fatores primordiais para uma sobrevida maior de pacientes com câncer de mama. $O$ tratamento rápido contribui para melhores condições de vida e menor ônus para o sistema de saúde.
\end{abstract}

Palavras-chave: neoplasias da mama, diagnóstico precoce, longevidade, fatores de tempo, prognóstico.

\section{CORRELATION BETWEEN TIME OF DIAGNOSIS, TREATMENT AND SURVIVAL IN PATIENTS WITH BREAST CANCER: A LITERATURE REVIEW}

\begin{abstract}
Breast cancer is the most common malignant tumor in women in the world. It is an important public health problem due mainly to its late diagnosis and high mortality. The objective was to perform a review of the literature, looking for studies that correlate the time elapsed between the diagnosis of breast cancer and the beginning of chemotherapy treatment. A bibliographic survey was carried out in the databases: Latin American and Caribbean Literature in Health Sciences (LILACS), PubMed, International Literature in Health Sciences (MEDLINE), Scientific Electronic Library Online (SciELO), Library Information System WHO (WHOLIS). The terms "breast cancer" AND "time of diagnosis" were used. The time of diagnosis and start of treatment are essential factors for a longer survival of patients with breast cancer. Rapid treatment contributes to better living conditions and lower costs for the health system.
\end{abstract}

Keywords: Breast neoplasms, early diagnosis, longevity, time factors, prognosis.

\section{INTRODUÇÃO}

O câncer de mama é o tumor maligno mais incidente em mulheres no mundo, incluindo os países mais desenvolvidos. Trata-se de um importante problema de saúde pública em virtude, principalmente, do seu diagnóstico tardio e, por conseguinte, alta mortalidade. Nos últimos anos, com a medicina moderna, tem-se observado um melhor prognóstico da doença em razão dos avanços cirúrgicos, medicamentosos e principalmente em decorrência do diagnóstico precoce $^{1}$.

Para a população em nosso país geralmente, o risco de ter câncer de mama ao longo da vida é da ordem de $8 \%$, ou seja, uma em cada doze mulheres desenvolverá câncer de mama ao longo da vida e este risco é tido como o risco basal da população do sexo feminino ${ }^{2}$. A 
recomendação do Ministério da Saúde - assim como a da Organização Mundial da Saúde e a de outros países - é a realização da mamografia de rastreamento (quando não há sinais nem sintomas) em mulheres de 50 a 69 anos, uma vez a cada dois anos. Já quando há suspeita, decorrente da anamnese e exame físico, se realiza a mamografia de diagnóstico ${ }^{3}$. Os sinais e sintomas mais comuns são: nódulo fixo, endurecido e, geralmente, indolor; pele da mama avermelhada, retraída ou parecida com casca de laranja; alterações no mamilo; pequenos nódulos na região dos braços (axilas) ou no pescoço; saída espontânea de líquido dos mamilos ${ }^{4}$. Por outro lado, a American Cancer Society e a Sociedade Brasileira de Mastologia orientam o início do rastreamento mamográfico aos 40 anos de idade, devido a alta taxa de incidência entre as mulheres de 40 a 50 anos.

É conhecido cientificamente que o atraso no diagnóstico e tratamento das neoplasias mamárias pode comprometer a sobrevida das pacientes portadoras da doença. No âmbito da assistência, a definição do tempo de atraso pode compreender três momentos distintos: o primeiro ocorre do início dos sintomas até a primeira consulta médica; o segundo compreende o período entre a primeira consulta até o primeiro acesso ao serviço de referência especializado; e o terceiro, da primeira avaliação no serviço especializado até o tratamento específico ${ }^{5}$. Recentemente, o Ministério da Saúde do Brasil determinou, por meio de portaria, que o tempo entre o registro do diagnóstico de câncer no prontuário médico e o início do tratamento não deve ultrapassar 60 dias $^{6}$.

Estudo realizado nos Estados Unidos (Carolina do Norte), com mulheres de baixa renda, evidenciou que uma em cada dez mulheres esperaram período maior ou igual a 60 dias para iniciar o tratamento após o diagnóstico, tendo sido esse intervalo associado com diminuição da sobrevida global em $66 \%$ e por câncer de mama em $85 \%$, entre pacientes com estadiamento avançado ${ }^{7}$. Outro estudo que avaliou o impacto da demora na realização de cirurgia com finalidade curativa, na sobrevida, concluiu que intervalos superiores a 12 semanas estão associados ao aumento da mortalidade pela doença ${ }^{8}$.

O câncer de mama é um problema de saúde pública no Brasil e em diversos países do mundo. A mortalidade por esta patologia é alta. Através do estudo busca-se a confirmação de que o período entre o diagnóstico e tratamento quimioterápico influencia na sobrevida de pacientes.

O objetivo deste trabalho é realizar uma revisão da literatura médica buscando estudos que correlacionem o tempo transcorrido entre o diagnóstico do câncer de mama e o início do tratamento quimioterápico.

\section{METODOLOGIA}

Realizou-se um levantamento bibliográfico nas bases de dados: Literatura Latino-Americana e do Caribe em Ciências da Saúde (LILACS), PubMed, Literatura Internacional em Ciências da Saúde (MEDLINE), Scientific Electronic Library Online (SciELO), Sistema de Informação da Biblioteca da OMS (WHOLIS). Foram utilizados os termos "breast cancer" AND "diagnostic time". A inclusão se deu por artigos em inglês e português com o tema específico deste trabalho dos últimos cinco anos. Todos os artigos que não se enquadrem nos termos especificados foram descartados.

\section{REVISÃO DE LITERATURA \\ Variabilidade socioeconômica}

Realizaram um estudo no qual se aplicou um questionário às pacientes que acompanhavam o câncer de mama no Hospital do Câncer de Pernambuco (HCP). Ficou demonstrado que um dos fatores relacionados ao tratamento em tempo adequado é o aspecto socioeconômico, pois a maioria das pacientes não possuía veículo próprio $(80 \%)$, havendo necessidade de espera de transporte por parte da esfera pública, ou mesmo, de amigos e familiares $^{9,10}$.

Em outro estudo, separaram a casuística em perfis: (A) de mulheres predominantemente brancas de maior nível de escolaridade, com tratamento custeado pela rede suplementar ou particular e média de idade de 56 anos; (B) de mulheres brancas, com predominância de escolaridade até 11 anos (54,9\%), tratamento custeado pelo SUS e média de 59 anos de idade; dois perfis ( $C$ e $D)$ incluindo mulheres predominantemente pardas, com predomínio de tratamento custeado pelo SUS e médias da idade de 52 e 55 anos, respectivamente; por fim, o perfil (E) de mulheres na sua totalidade de raça/cor preta, com predominância de escolaridade $<8$ anos, tratamento custeado pelo SUS e média de 59 anos de idade. A maioria das mulheres incluídas no perfil A $(86,5 \%)$ e no perfil 
B $(53,7 \%)$ apresentaram intervalo entre diagnóstico e início de tratamento $\leq 60$ dias. Menos da metade das mulheres dos perfis $C$ $(43,6 \%)$, D $(43,4 \%)$ e E $(36,1 \%)$ iniciaram tratamento neste intervalo, sendo que no perfil $\mathrm{E}$ $41,7 \%$ iniciaram o tratamento em intervalo $\geq 91$ dias (valor de $\mathrm{p}<0,05)$. A proporção de mulheres com estágios III e IV, no momento do diagnóstico, foi significativamente maior nos perfis $C(48,1 \%)$, $D(45,3 \%)$ e $E(50,0 \%)$, enquanto que a proporção de mulheres com câncer nos estágios iniciais, In situ e I, foram mais frequentes no perfil A $(44,4 \%)^{11}$. Esses dados indicam que as desigualdades socioeconômicas tiveram influência nos estágios de câncer de mama das pacientes e, por sua vez, na qualidade de tratamento oferecido.

Em estudo multicêntrico, encontraram que pacientes do Sistema Único de Saúde (SUS) apresentaram maiores proporções de doença avançada ao diagnóstico $(\mathrm{P}<0,001)$ e menor sobrevida nos estágios III e IV do câncer ( $\mathrm{P}<0,002$ e $\mathrm{P}<0,008$ respectivamente) quando comparadas às mulheres com cobertura de serviços privados $^{12}$.

Desigualdades sociais - ou aquelas desigualdades que, quando associadas a características individuais como escolaridade, renda, etnia entre outras colocam alguns grupos em desvantagem em relação a outro - podem resultar não só em piores condições de saúde como também em desigualdades no acesso e utilização de serviços ${ }^{2}$.

Nota-se que aspectos relacionados às condições socioeconômicas de pacientes têm profunda relevância no diagnóstico precoce e tratamento dos mesmos. Quanto mais desfavorecidos economicamente, normalmente, pior é o prognóstico e tempo de tratamento mais demorado em virtude de atrasos no sistema de saúde ou mesmo demora na procura de atendimento. Pessoas com melhores condições financeiras acabam sendo diagnosticadas e tratadas mais precocemente e, por sua vez, seu prognóstico acaba sendo melhor.

A cultura de desinformação também é prejudicial, ao passo que, indivíduos sem acesso à educação de qualidade, não sabem observar lesões neoplásicas como atípicas e procurar ajuda mais rapidamente.

\section{Demora para realização do diagnóstico}

Quanto ao tempo de procura por auxílio médico, várias questões podem ser atribuídas, como a demora em notar o aparecimento dos sintomas; dificuldade de acesso aos serviços e problemas no referenciamento do paciente para o serviço adequado. Foi descrito que retardos de três a seis meses estiveram associados a uma pior sobrevida das pacientes ${ }^{10,13,14}$.

\section{Atraso no tratamento}

Em estudo realizado em 2016, avaliaram 273.521 pacientes receptores estrogêniopositivos, observando que, cada mês adicional de atraso no tratamento reduziu o resultado da quimioterapia em $11,1 \%{ }^{15}$.

Em pesquisa de 2018 evidenciaram que, em tumores com receptores positivos para HER2, a sobrevida global foi afetada pelo tempo de tratamento com quimioterapia ${ }^{16}$.

Outro estudo analisando o intervalo de tempo entre a cirurgia e radioterapia (até 6 semanas) foi associado a uma razão de risco de 1,19 (95\% intervalo de confiança $[\mathrm{Cl}]$ 1,01-1,39, $\mathrm{p}=0,004)$ para recorrência local, com aumento de $0,5 \%$ no risco de recorrência local por dia ${ }^{17}$.

$\mathrm{Na}$ literatura é consenso que quanto menor o intervalo entre diagnóstico e tratamento, melhor o prognóstico e maior a sobrevida da paciente. Nos estágios mais avançados a intervenção rápida é fundamental para a eficácia do tratamento ou, no caso de cuidados paliativos, para o conforto do paciente.

\section{Fatores prognósticos do câncer de mama}

Diagnosticado e tratado oportunamente, o câncer de mama se revela um tumor de bom prognóstico e a sobrevida em cinco anos chega a $85 \%$. Contudo, em países em desenvolvimento, as taxas de mortalidade são mais elevadas. No Brasil, a alta taxa de mortalidade pode ser explicada parcialmente pelo fato de, em média, $60 \%$ dos tumores de mama serem diagnosticados em estádios avançados ${ }^{9}$.

O elevado tempo de espera para a realização dos exames diagnósticos e para o início do tratamento podem produzir consequências graves para as pacientes, como a diminuição das suas chances de cura e a taxa de sobrevida. Além disso, um tratamento realizado tardiamente traz prejuízos à qualidade de vida, pois requer abordagens mais agressivas, necessidade de utilização de múltiplas modalidades terapêuticas, e resulta na sobreposição de sequelas. É importante considerar ainda o aumento dos gastos públicos como consequência dos tratamentos mais caros e prolongados, bem como os custos 
previdenciários decorrentes do afastamento do trabalho. ${ }^{6}$.

Em conclusão, o tempo de diagnóstico e início do tratamento é fator primordial para uma sobrevida maior de pacientes com câncer de mama. Fazem-se necessárias mais ações públicas de conscientização acerca desta neoplasia, bem como maior número de serviços que possam fazer o diagnóstico mais rápido. Ademais, o tratamento rápido contribui para melhores condições de vida e menor ônus para o sistema de saúde, uma vez que serão menos agressivos e recidivantes.

\section{DECLARAÇÃO DE CONFLITO DE INTERESSES}

Os autores declaram não haver qualquer potencial conflito de interesse que possa interferir na imparcialidade deste trabalho científico.

\section{REFERÊNCIAS}

1. Falcetta FS, Träsel HDAV, Almeida FK, Falcetta MRR, Falavigna M, Rosa DD. Effects of physical exercise after treatment of early breast cancer: systematic review and meta-analysis. Breast Cancer Res Treat. 2018;170(3):455-76. DOI: https://doi.org/10.1007/s10549-018-4786-y

2. Barata RB. Como e por que as desigualdades sociais fazem mal à saúde. Rio de Janeiro: Editora Fiocruz; 2009.

3. Instituto Nacional do Câncer José de Alencar Costa da Silva - Inca (Brasil). Câncer de Mama. [acesso em 23 abr 2019]. Disponível em: http://www.inca.org.br

4. The American College of Radiology - ACR (EUA). ACR BI-RADS Fifth Edition. [acesso em 24 abr 2019]. Disponível em: https://www.acr.org//media/ACR/Files/RADS/BI-RADS/BIRADS-

Reference- Card.pdf

5. Coates AS. Breast cancer: delays, dilemmas, and delusions. Lancet. 1999;353(9159): 1112-3.

6. Ministério da Saúde (Brasil). Portaria $n^{\circ} 876$, de 16 de maio de 2011. Diário Oficial da União 2013; 17 mai 2011.

7. McLaughlin JM, Anderson RT, Ferketich AK, Seiber EE, Balkrishnan R, Paskett ED. Effect on survival of longer intervals between confirmed diagnosis and treatment initiation among low- income women with breast cancer. J Clin Oncol. 2012;30(36):4493-500.

DOI:

https://doi.org/10.1200/JCO.2012.39.7695

8. Shin D W, Cho J, Kim SY, Guallar E, Hwang SS, Cho B, Park JH. Delay to curative surgery greater than 12 weeks is associated with increased mortality in patients with colorectal and breast cancer but not lung or thyroid cancer. Ann Surg Oncol. 2013;20(8):2468-76. DOI: https://doi.org/10.1245/s10434-013-2957-y

9. Paiva CJK, Cesse EÂP. Aspectos relacionados ao atraso no diagnóstico e tratamento do câncer de mama em uma unidade hospitalar de Pernambuco. Rev Bras Cancerol. 2015;61(1):2330. DOI: https://doi.org/10.32635/21769745.RBC.2015v61n1.374

10. Montella M, Crispo A, D'aiuto G, Marco M, Bellis G, Fabbrocini G, Silvesrtra P. Determinant factors for diagnostic delay in operable breast cancer patients. Eur J Cancer Prev. 2001;10(1):539. DOI: https://doi.org/10.1097/00008469200102000-00006

11. Cabral ALLV, Giatti L, Casale C, Cherchiglia ML. Vulnerabilidade social e câncer de mama: diferenciais no intervalo entre o diagnóstico e o tratamento em mulheres de diferentes perfis sociodemográficos. Ciên Saúde Col. 2019;24(1):613-22. DOI: https://doi.org/10.1590/1413$\underline{81232018242.31672016}$

12. Liedke PE, Finkelstein DM, Szymonifka J, Barrios $\mathrm{CH}$, Chavarri-Guerra Y, Bines J, Goss PE. Outcomes of breast cancer in Brazil related to health care coverage: a retrospective cohort study. Cancer Epidemiol Prev Biomarkers. 2014;23(1):126-33. DOI: https://doi.org/10.1158/1055-9965.EPI-13-0693

13. Richards MA, Westcombe AM, Love SB, Littlejohns $P$, Ramirez AJ. Influence of delay on survival in patients with breast cancer: a systematic review. Lancet. 1999;353(9159):111926.

14. Ramirez AJ, Westcombe AM, Burgess CC, Sutton S, Littlejohns P, Richards MA. Factors predicting delayed presentation of symptomatic breast cancer: a systematic review. Lancet. 1999;353(9159):1127-31. 
15. Chavez-MacGregor M, Clarke CA, Lichtensztajn DY, Giordano, S. H. Delayed initiation of adjuvant chemotherapy among patients with breast cancer. JAMA Oncol. 2016;2(3):322-9.

DOI:

https://doi.org/10.1001/jamaoncol.2015.3856

16. Mateo AM, Mazor AM, Obeid E, Sigurdson ER, DeMora L, Handorf EA, Bleicher RJ. Time to surgery and the impact of delay on triple negative breast cancers and other phenotypes. J Clin Oncol. 2018;36(15 suppl):12606. DOI: https://doi.org/10.1200/JCO.2018.36.15 suppl.e $\underline{12606}$

17. Punglia RS, Saito AM, Neville BA, Earle CC, Weeks JC. Impact of interval from breast conserving surgery to radiotherapy on local recurrence in older women with breast cancer: retrospective cohort analysis. BMJ. 2010;340:c845.

DOI: https://doi.org/10.1136/bmj.c845

18. Instituto Nacional do Câncer José de Alencar Costa da Silva - Inca (Brasil). Controle do câncer de mama - documento de consenso. [acesso em 24 abr 2019]. Disponível em: http://www.inca.gov.br/publicacoes/consensoint egra.pdf

19. Secretaria de Saúde do Estado do Paraná (Brasil). Programa do INCA - Parte VI (Câncer de mama). [acesso em 24 abr 2019]. Disponível em: http://www.saude.pr.gov.br/modules/conteudo/ print.php?conteudo $=268$

20. Sainsbury JR, Johnston C, Haward B. Effect on survival of delays in referral of patients with breast-cancer symptoms: a retrospective analysis. Lancet. 1999;353(9159):1132-5.

21. Bish A, Ramirez A, Burgess C, Hunter M. Understanding why women delay in seeking help for breast cancer symptoms. J Psychos Res. 2005;58(4):321-6. DOI: https://doi.org/10.1016/i.jpsychores.2004.10.007

22. Tribunal de Contas da União (Brasil). Secretaria de Fiscalização e Avaliação de Programas do Governo. Relatório de auditoria operacional na Política Nacional de Atenção Oncológica. Brasília: TCU; 2011.
23. Traldi M C, Galvão P, Morais SSD, Fonseca MRCDC. Demora no diagnóstico de câncer de mama de mulheres atendidas no Sistema Público de Saúde. Cad Saúde Col. 2016;24(2):185-91. DOI: https://doi.org/10.1590/1414$\underline{462 \times 201600020026}$

24. Zhan $\mathrm{QH}$, Fu JQ, Fu FM, Zhang J, Wang C. Survival and time to initiation of adjuvant chemotherapy among breast cancer patients: a systematic review and metaanalysis. Oncotarget. 2018;9(2):2739-51. DOI: https://doi.org/10.18632/oncotarget.23086

25. Trufelli DC, Matos LLD, Santi PX, Del Gigli, A. Adjuvant treatment delay in breast cancer patients. Rev Assoc Méd Bras. 2015;61(5):411-6. DOI: $\quad$ https://doi.org/10.1590/1806$\underline{9282.61 .05 .411}$

26. Trufelli DC, Miranda VDC, Santos MBB. D., Fraile, N. M. P., Pecoroni, P. G., Gonzaga, S. D. F. R., \& Del Giglio, A. Análise do atraso no diagnóstico e tratamento do câncer de mama em um hospital público. Rev Assoc Méd Bras. 2008;54(1):72-6. DOI: https://doi.org/10.1590/S0104$\underline{42302008000100024}$

27. Instituto Nacional de Câncer José Alencar Gomes da Silva -INCA (Brasil). Controle do Câncer de Mama, Documento de Consenso. Rio de Janeiro: INCA; 2004. 